\title{
RESISTÊNCIA DE ISCAS GRANULADAS, DISTRIBUÍDAS A GRANEL E EM MICROPORTA-ISCAS, À AÇÃO DA UMIDADE EM PLANTIO DE Pinus taeda NO PLANALTO SUL-CATARINENSE
}

\author{
RESISTANCE OF BAIT GRANULATED DISTRIBUTED IN BULK AND BAIT HOLDER TO \\ ACTION OF HUMIDITY IN AREAS OF TO ACTION OF MOISTURE IN Pinus taeda PLANTING IN \\ SOUTHERN HIGHLANDS CATARINENSE
}

David Alexandre Buratto ${ }^{1}$ Nilton José Sousa ${ }^{2}$ Marcelo Dias Souza ${ }^{1}$ Flávio Augusto Rolin ${ }^{3}$

\begin{abstract}
RESUMO
Os programas de controle para formigas-cortadeiras do gênero Acromyrmex na região sul do Brasil são pouco estudados, tendo suas informações embasadas no controle do gênero Atta e aos danos causados por formigas-cortadeiras em plantios de Eucalyptus spp. Esta falta de informações sobre controle de formigas-cortadeiras leva algumas empresas florestais de Santa Catarina à repetição de padrões que não são adequados às infestações e às espécies de formigas-cortadeiras que ocorrem neste Estado. Com isso, ocorre uma distribuição inadequada de iscas granuladas a granel, nas quatro estações do ano, que acabam degradadas pelo excesso de umidade provocado pela neblina e pelas precipitações constantes que ocorrem na região. Diante deste quadro, os experimentos deste trabalho tiveram como objetivos: identificar as espécies de formigas-cortadeiras; determinar a densidade de formigueiros por unidade área; analisar e avaliar a influência das estações do ano e da precipitação em cada estação sobre a conservação e degradação das iscas granuladas à base de sulfluramida $(0,3 \%)$ distribuídas a granel e na forma de microporta-iscas. Para tanto, foram instalados experimentos na Fazenda Dois Irmãos, de propriedade da empresa Florestal Rio Marombas Ltda., em áreas de plantios de Pinus taeda L., localizados no município de São Cristóvão do Sul, no planalto sul do estado de Santa Catarina. Concluiu-se que: A única espécie de formiga-cortadeira encontrada na área avaliada é Acromyrmex crassispinus (FOREL, 1909). Na área experimental encontramse 17 formigueiros, a média é de 0,94 formigueiros por hectare, com área aparente menor que $1 \mathrm{~m}^{2}$ cada um; a umidade da região do planalto sul-catarinense degrada as iscas granuladas distribuídas a granel; as iscas granuladas distribuídas a granel permanecem conservadas sete dias na primavera e três dias nas demais estações do ano; as iscas granuladas distribuídas em microporta-iscas permanecem conservadas 15 dias no verão e 30 dias nas demais estações do ano; os microporta-iscas apresentam maior resistência à umidade no campo em relação às iscas granuladas distribuídas a granel; a abertura dos microporta-iscas, provocada pelo consumo parcial das iscas, que é uma característica das formigas Acromyrmex spp., acelera a deterioração das iscas restantes em seu interior.
\end{abstract}

Palavras-chave: Acromyrmex; sulfluramida; formiga-cortadeira.

\begin{abstract}
Control programs for leaf-cutting ants of the genus Acromyrmex in southern Brazil are poorly studied and the information comes from the control of the genus Atta and the damage caused by leaf-cutting ants in Eucalyptus spp. This lack of information about this ant makes some forest companies of Santa Catarina

1 Engenheiro Florestal, Doutorando em Engenharia Florestal, Universidade Federal do Paraná, CIFLOMA, Campus III, Av. Pref. Lothário Meissner, 632, Jardim Botânico, CEP 80210-170, Curitiba (PR), Brasil. davidburatto@yahoo.com.br / marcelo.dias@florestal.eng.br

2 Engenheiro Florestal, Dr., Professor do Departamento de Ciências Florestais, Universidade Federal do Paraná, CIFLOMA, Campus III, Av. Pref. Lothário Meissner, 632, Jardim Botânico, CEP 80210-170, Curitiba (PR), Brasil. nilton.ufpr@gmail.com

3 Engenheiro Florestal, Mestre em Engenharia Florestal, Rua José Salustiano Lara, 58, Betim (MG), Brasil. flaviorolim11@gmail.com
\end{abstract}

Recebido para publicação em 10/01/2015 e aceito em 13/01/2016

Ci. Fl., v. 27, n. 3, jul.-set., 2017 
repeat patterns that are not suitable for infestations and for the species of leaf-cutting ants that occur in this state. Along with this, an inadequate distribution of bulk granular baits, in the four seasons, which end up degraded by excessive moisture caused by fog and by constant rainfall occurring in the region. Given this context, the experiments of this work had the following objectives: identify the species of leaf-cutting ants; determine the nest density per unit area; analyze and evaluate the influence of the year and the rainfall stations in each season, about the conservation and degradation of granulated baits based on sulfluramide $(0.3 \%)$ in bulk and distributed in the form of bait holder. To do so, experiments in Dois Irmãos Farm, owned by Florestal Rio Marombas company in areas of Pinus taeda L., located in the municipality of São Cristóvão do Sul, in the southern highlands of Santa Catarina state were installed. It was concluded that: the only species found in the leaf-cutting ant Acromyrmex crassispinus area is assessed (FOREL, 1909). At the experimental site there are 17 nests, the average is 0.94 nests per hectare, less than $1 \mathrm{~m}^{2}$ each apparent area; moisture from southern Santa Catarina plateau region degrades the granulated baits distributed in bulk; the granulated baits distributed in bulk remain conserved seven days in the spring and three days in other seasons; the granulated baits distributed in bait holder remain preserved 15 days in the summer and 30 days in other seasons; the bait holder presents greater resistance to moisture in the field in relation to granulated baits distributed in bulk; the opening of the bait holder, caused by partial consumption of the bait, which is a feature of ants Acromyrmex spp., accelerates the deterioration of the remaining baits inside it.

Keywords: Acromyrmex; sulfluramid; leaf-cutting ants.

\section{INTRODUÇÃO}

Uma das peculiaridades mirmecológicas da região sul do Brasil, principalmente em reflorestamentos de pinus, é a predominância de formigas-cortadeiras pertencentes ao gênero Acromyrmex, também conhecidas como quenquém (PACHECO; REIS; BRIDI, 2001).

A espécie Acromyrmex crassispinus distribui-se na Argentina, Brasil, Paraguai e Uruguai (HÖLLDOBLER; WILSON, 1990; MAYHÉ-NUNES, 1991). No Brasil ocorre em São Paulo, Rio de Janeiro, Paraná, Santa Catarina, Rio Grande do Sul, Distrito Federal e Minas Gerais (GONÇALVES, 1961; MAYHÉ-NUNES, 1991; DELLA LUCIA; VILELA, 1993). Na região sul do Brasil é a espécie de formigacortadeira mais comum (GONÇALVES, 1961; DELLA LUCIA; VILELA, 1993; RANDO; FORTI, 2005) e nos plantios de Pinus taeda localizados no norte de Santa Catarina, no entanto, são poucas as pesquisas feitas sobre essa espécie (REIS FILHO; OLIVEIRA, 2002).

Segundo Mayhé-Nunes (1991), Acromyrmex crassispinus apresenta espinhos mesonotais anteriores aproximadamente do tamanho dos pronotais laterais, às vezes um pouco maiores ou menores; espinhos pronotais inferiores curvados para baixo ou para trás; tubérculos do tergo I do gáster numerosos e bem desenvolvidos, aguçados e distribuídos ao acaso; pilosidade fina e semiereta no pronoto e coloração castanho-enegrecida ou negra.

As formigas-cortadeiras, Atta spp. (saúva) e Acromyrmex spp. (quenquém), causam danos significativos às plantações florestais, especialmente durante a implantação dos povoamentos, pois as mudas são intensamente atacadas e, muitas vezes, é preciso replantar as áreas. As saúvas, causam danos mais expressivos, por esse motivo, as técnicas de controle, na sua maioria, são desenvolvidas para este gênero e posteriormente adaptadas para o controle de quenquéns.

As adaptações dessas técnicas de controle, com frequência, são feitas sem critérios adequados, especialmente no caso do uso de iscas granuladas. Dessa forma, a reprodução do padrão de distribuição de iscas a granel é uma prática comum em vários Estados que têm predominância de Atta spp. como São Paulo e Minas Gerais. Porém, nestes Estados, o clima é mais seco e as chuvas são mais definidas, fato que não se repete na região sul, em que Acromyrmex spp. é abundante, a umidade é alta e as chuvas são constantes.

A grande vantagem das iscas granuladas é a forma como podem ser distribuídas no campo, podendo ser aplicadas diretamente nos formigueiros ou distribuídas sistematicamente, utilizando saquinhos de polietileno, conhecidos como microporta-iscas (ZANUNCIO et al., 1992). Para a isca granulada ser eficiente, segundo Cherrettl et al. (1973), apud Della Lúcia et al. (1993), esta deve ser resistente às intempéries climáticas como umidade e elevadas temperaturas, para se manter atrativa às formigas.

A sulfluramida foi desenvolvida nos Estados Unidos como uma substância, para controlar formigas 
e baratas em residências, pertence ao grupo químico das sulfonamidas fluoroalifáticas. Seu modo de ação é por ingestão e a composição das iscas apresenta $0,3 \%$ de ingrediente ativo e 99,7\% de atrativos e material inerte. O produto tem $42,59 \%$ de biodegradação em 28 dias, à biodegradabilidade total no solo ocorre entre 90 e 120 dias (SOUSA, 1996). Os resultados apresentados pelas iscas à base de sulfluramida são excelentes e comparáveis aos apresentados por iscas à base de dodecacloro (ZANUNCIO et al., 1992).

Segundo Juruena (1980), as iscas granuladas são ideais para o controle de formigas-cortadeiras, pois são de fácil aplicação, dispensam aparelhos e não apresentam perigos de intoxicação que o manejo de outros tipos de formicidas causam, além de apresentarem um alto grau de eficiência.

Uma das principais vantagens do uso de porta-iscas é que os formigueiros não precisam necessariamente ser localizados, dispensando mão de obra, pois, inverte-se o processo, as formigas localizam os porta-iscas devido a sua atratividade (ALMEIDA; ALVES, 1982)

Os microporta-iscas, com 5 a 10 gramas de iscas, têm maior probabilidade teórica de sucesso no controle de quenquéns, pois apresentam uma nuvem de pontos de distribuição maior do que os outros tipos de recipientes. Porém, deve ser lembrado que os microporta-iscas também apresentam desvantagens, sendo a principal dificuldade determinar antes da aplicação a densidade mais adequada para cada área, proporcionando uma quantidade de iscas sem excessos, que seja eficaz para eliminar os formigueiros. Os autores que avaliaram este tipo de porta-iscas observaram que o uso do microporta-iscas, com quantidade maior de isca certamente implicariam em um aumento do consumo parcial, causado principalmente pelas formigas do gênero Acromyrmex (LARANJEIRO et al., 1986).

A exposição da isca granulada às intempéries climáticas, como chuva, umidade do ar e orvalho, deterioram-nas rapidamente, fazendo com que percam a atratividade para as formigas-cortadeiras. As iscas granuladas distribuídas a granel podem ficar deterioradas em poucas horas, normalmente muito antes de serem localizadas pelas formigas-cortadeiras (BURATTO, 2013). Para a isca granulada ser eficiente, esta deve ser resistente às intempéries climáticas como umidade e elevadas temperaturas, para se manter atrativa às formigas (CHERRETT et al., 1973).

Para amenizar esse problema, empresas florestais da região sul do Brasil, na qual predominam Acromyrmex spp, têm investido em pesquisas para seu controle, pois pouco se conhece sobre a distribuição e comportamento desse gênero em áreas de Pinus spp. no sul do Brasil, bem como sobre a melhor forma de distribuição de iscas granuladas.

Com base nestas premissas, este trabalho teve como objetivos: analisar e avaliar a influência das estações do ano e da precipitação em cada estação, sobre a resistência das iscas granuladas à base de sulfluramida $(0,3 \%)$ distribuídas a granel e na forma de microporta-iscas.

\section{MATERIAL E MÉTODOS}

O experimento deste trabalho foi instalado no talhão 10 (50¹8'14'W e $27^{\circ} 16^{\prime} 39^{\prime}$ 'S), da Fazenda Dois Irmãos, de propriedade da empresa Florestal Rio Marombas Ltda., localizada no planalto sulcatarinense no município de São Cristóvão do Sul, distante aproximadamente $300 \mathrm{~km}$ de Florianópolis, capital do Estado, a $1.160 \mathrm{~m}$ de altitude. A área do talhão 10 possuía 18 ha, dos quais foram utilizados 1,4 ha para a instalação dos experimentos deste trabalho.

De acordo com a classificação de Köppen, o município de São Cristóvão do Sul tem clima temperado com chuvas bem distribuídas ao longo do ano. O clima é classificado como mesotérmico úmido com invernos frios e verões amenos $(\mathrm{Cfb})$, apresentando temperatura média anual de $13^{\circ} \mathrm{C}$, variando em média de $9^{\circ} \mathrm{C}$ no inverno e $18^{\circ} \mathrm{C}$ no verão (EMBRAPA, 1988).

A Fazenda Dois Irmãos está situada na região bioclimática I de Santa Catarina, com altitudes que variam de 600 a 1300 metros. A vegetação da região é caracterizada como Floresta Ombrófila Mista, a temperatura média anual da região é de 12 a $19^{\circ} \mathrm{C}$, com médias das temperaturas mínimas no mês mais frio, variando de 5 a $8^{\circ} \mathrm{C}$. A temperatura máxima no mês mais quente oscila entre 22 a $31^{\circ} \mathrm{C}$. A mínima absoluta na região foi de $-12^{\circ} \mathrm{C}$, a precipitação anual oscila entre 1.300 a $2.400 \mathrm{~mm}$, sem deficit hídrico (EMBRAPA, 1988).

As iscas granuladas testadas tinham como ingrediente ativo a molécula Sulfluramida (N-ethylperfluoro-octane-1-sulfonamede: $3 \mathrm{~g} / \mathrm{kg}$, inseticida do grupo das sulfonamidas fluoroalifáticas). 
Estas foram distribuídas no campo de duas formas: a granel; e embaladas na forma de microporta-iscas (saquinho de polietileno, com dimensões aproximadas de $73 \mathrm{~mm}$ x $61 \mathrm{~mm}$ x 0,06 $\mathrm{mm}$ de espessura sem isca granulada e $4 \mathrm{~mm}$ de espessura com isca granulada) de $5 \mathrm{~g}$.

Para a implantação do experimento foram instalados quatro blocos, com dimensões de $50 \mathrm{~m} \times 70$ $\mathrm{m}$. Cada um possuía uma área total de $3.500 \mathrm{~m}^{2}$. Os blocos foram divididos em oito parcelas (tratamentos) de $200 \mathrm{~m}^{2}$ cada, distantes $10 \mathrm{~m}$ uma da outra, para receberem os tratamentos com isca aplicada a granel e na forma de microporta-iscas. Sendo assim, o experimento foi instalado em quatro blocos e dividido em 32 parcelas. Foi mantida uma distância de $20 \mathrm{~m}$ entre blocos e dentro de cada bloco uma área de segurança de $200 \mathrm{~m}^{2} \mathrm{em}$ volta de cada parcela (Figura 1).

Depois que os blocos e parcelas estavam fisicamente instalados em campo, a área destes foi percorrida, seguindo a linha de tocos do povoamento anterior. Todos os formigueiros encontrados foram numerados, medidos (maior comprimento e largura, com o auxílio de uma trena) e fotografados. Também foram coletadas informações sobre a composição superficial.

De cada formigueiro localizado, foram coletados 10 indivíduos que foram acondicionados em frascos contendo álcool $70 \%$, posteriormente todo material coletado foi encaminhado ao Laboratório de Proteção Florestal da Universidade Federal do Paraná, no qual os exemplares foram montados e etiquetados. Após a montagem, os exemplares foram enviados à Universidade de São Paulo/Campus Pirassununga - SP, aos cuidados do Dr. Pedro Pacheco dos Santos Lima, que procedeu a identificação do material.

Em seguida, os tratamentos foram distribuídos por meio de sorteio dentro de cada bloco. Cada bloco recebeu oito tratamentos, sendo quatro com isca a granel e quatro com microporta-iscas (Tabela 1).

Na primavera, este experimento teve inicio no dia 31/10/2011, no verão em 30/01/2012, no outono teve inicio no dia 02/04/2012 e no inverno em 02/07/2012. Nas avaliações realizadas $(1,2,3,7,15,30$ 45 dias após a distribuição de iscas granuladas) foi analisado o estado de conservação da isca distribuída a granel e na forma de microporta-iscas. As avaliações foram realizadas duas vezes ao dia, em todas as avaliações, a primeira de manhã, às $8 \mathrm{~h} 00$ e a segunda à tarde, às $16 \mathrm{~h} 00$.

Nestas observações era determinado de forma visual se existiam modificações físicas que podiam inutilizar as iscas granuladas distribuídas a granel ou os grânulos dentro dos microporta-iscas. Para tanto, foram observados três parâmetros: Isca Sem Deterioração (SD), ou seja, intacta, com todas as suas características preservadas (tamanho, e dureza); Isca Parcialmente Deteriorada (PD), iscas granuladas apresentavam mudanças na forma física, se tornando inchadas ou esfareladas; e Isca Totalmente Deteriorada (TD), as iscas granuladas formavam uma massa ou não se encontravam mais no ponto de distribuição.

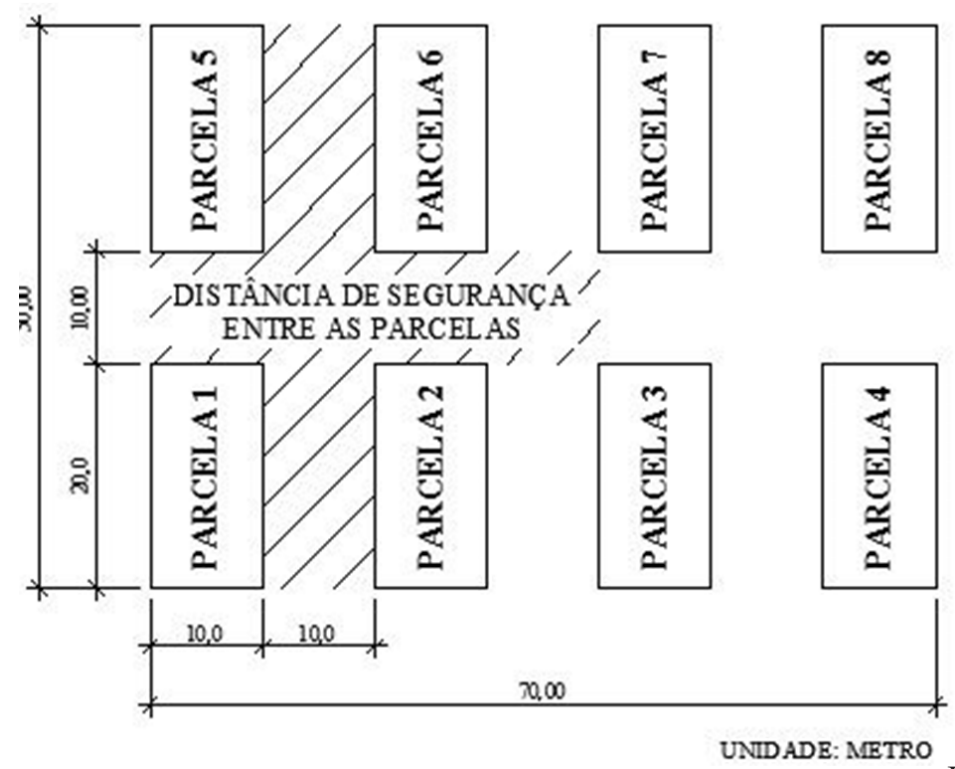

Fonte: $\mathrm{O}$ autor.

FIGURA 1: Croqui de um bloco experimental instalado.

FIGURE 1: Sketch of an experimental block installed. 
TABELA 1: Distribuição dos tratamentos por meio de sorteio dentro de cada bloco.

TABLE 1: Distribution of treatments by lot within each block.

\begin{tabular}{ccccc}
\hline PARCELA & BLOCO 1 & BLOCO 2 & BLOCO 3 & BLOCO 4 \\
\hline 1 & T2G & T2G & T3M & T1G \\
2 & T4M & T3M & T2G & T2M \\
3 & T4G & T1M & T4M & T3M \\
4 & T1G & T4M & T1G & T1M \\
5 & T2M & T3G & T3G & T4G \\
6 & T3M & T4G & T2M & T2G \\
7 & T3G & T2M & T1M & T3G \\
8 & T1M & T1G & T4G & T4M \\
\hline
\end{tabular}

Em que: T: tratamento; 1 a 4: número do tratamento; G: granel e M: microporta-isca (Fonte: $\mathrm{O}$ autor).

Também se avaliou o consumo de isca granulada pelas formigas-cortadeiras. Parâmetro que foi observado apenas na primavera e no verão, pois considerou-se que a distribuição na primavera poderia controlar os formigueiros que já estavam presentes na área. A distribuição no verão visou ao controle dos formigueiros que ingressaram na área a partir da revoada que poderia ter ocorrido entre outubro e dezembro (primavera).

Para padronizar a distribuição nos tratamentos, as iscas (granel e microporta-isca) foram distribuídas seguindo-se as linhas de plantio de maneira sistemática em cada parcela, contendo $5 \mathrm{~g}$ de isca em cada ponto, com o objetivo de obter a melhor cobertura da área. Cada tratamento recebeu uma quantidade diferente de pontos de distribuição de isca, correspondendo à quantidade de iscas por parcela (repetição) que cada uma representou (Figura 2).

Para a distribuição dos tratamentos com isca a granel foi utilizado um dosador comumente usado na região e chamado popularmente de "cachimbo". Este dosador foi medido e padronizado para a quantidade exata de $5 \mathrm{~g}$ de isca granulada por ponto de distribuição. Os tratamentos com microporta-isca com $5 \mathrm{~g}$,

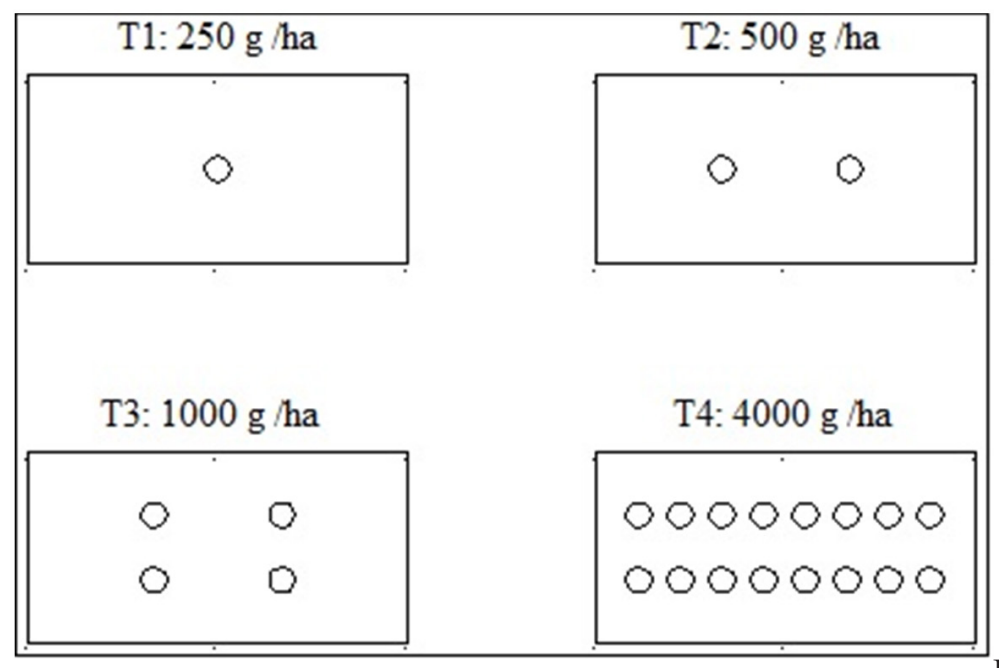

Fonte: $\mathrm{O}$ autor.

FIGURA 2:Esquema dos pontos de iscas granuladas distribuídas a granel (5 g) e forma de microporta-isca $(5 \mathrm{~g})$ nas parcelas.

FIGURE 2: Scheme granular baits points distributed in bulk $(5 \mathrm{~g})$ and the form of bait holder $(5 \mathrm{~g})$ in portions. 
seguiram o mesmo padrão de distribuição sistemático descrito para a distribuição das iscas a granel.

Cada ponto de distribuição foi demarcado com um piquete de madeira com $1 \mathrm{~m}$ de comprimento, a fim de facilitar a localização das iscas colocadas em cada parcela.

Consideraram-se as condições climáticas no período das avaliações a partir dos dados da estação meteorológica $\left(50^{\circ} 23^{\prime} 13\right.$ " W e $26^{\circ} 53^{\prime} 33^{\prime}$ ' S), situada na sede da empresa Florestal Rio Marombas Ltda., na BR-116, km 161, Fazenda Rio das Pedras, Ponte Alta do Norte, Santa Catarina, Brasil, distante aproximadamente $23 \mathrm{~km}$ da área experimental.

O delineamento experimental foi em blocos casualizados com oito tratamentos e quatro blocos, das quais os dados foram submetidos à análise de variância (ANOVA), em que se rejeitando H0, as médias foram comparadas pelo teste de Tukey em nível de $5 \%$ de probabilidade de erro. A variável resposta correspondeu ao percentual de deterioração, das quais passaram pelo teste de normalidade (Kolmogorov D: Normal test) e homogeneidade de variância (Bartlett) e transformados pela fórmula: $\operatorname{arcsen} \sqrt{ }(\mathrm{X} / 100)$, conforme recomendando por Banzatto e Kronka (2006), podendo garantir a normalidade dos dados. Os cálculos estatísticos foram efetuados por meio do software computacional SISVAR 5.1 (FERREIRA, 2008).

\section{RESULTADOS E DISCUSSÃO}

Nos quatro blocos amostrados foram encontrados 17 formigueiros, com área aparente menor que $1 \mathrm{~m}^{2}$ cada um. A média geral foi 0,94 formigueiros por hectare, todos eles eram da espécie Acromyrmex crassispinus (FOREL, 1909) (Hymenoptera: Formicidae: Myrmicinae), popularmente conhecida como quenquém-de-cisco. A ocorrência dessa espécie no interior de Santa Catarina, está de acordo com as citações de Gonçalves (1957), Della Lúcia e Vilela (1993), Reis Filho e Oliveira (2002), Nickele (2008) e Buratto et al. (2012).

\section{Influência da umidade sobre iscas granuladas distribuídas a granel}

Em relação às estações do ano, observou-se que na primavera e no outono as iscas permaneceram sem deterioração por mais tempo no campo (até a $3^{\mathrm{a}}$ e $4^{\mathrm{a}}$ avaliação, Figuras $3 \mathrm{~A} \mathrm{e} 3 \mathrm{C}$ ). No inverno, as iscas distribuídas a granel começaram a ser deterioradas mais rapidamente que nas demais estações (Figura 3D), isso comprova que há diferença entre a resistência da isca granulada no campo, quando estas são aplicadas em diferentes períodos do ano, ou seja, no inverno a probabilidade destas iscas deteriorarem é maior, tornando as iscas atrativas por um menor período.

$\mathrm{Na}$ primavera, as iscas granuladas de todos os tratamentos tiveram o mesmo comportamento, as iscas foram sendo deterioradas gradualmente, conforme foi aumentando a precipitação (Figura 3A).

No verão, na $4^{\mathrm{a}}$ avaliação, a precipitação acumulada foi de $70 \mathrm{~mm}$, assim, na $5^{\mathrm{a}}$ avaliação, as iscas granuladas de todos os tratamentos estavam deterioradas (Figura 3B).

No outono, as iscas granuladas do tratamento T2 (500 g/ha) foram deterioradas com o início da precipitação registrada na $3^{\text {a }}$ avaliação, as iscas dos demais tratamentos permaneceram conservadas, mas com o aumento da precipitação nas avaliações seguintes, as iscas granuladas de todos os tratamentos foram totalmente deterioradas (Figura 3C).

No inverno, nota-se que a deterioração das iscas granuladas iniciou com a precipitação observada entre a $3^{\mathrm{a}}$ e $4^{\mathrm{a}}$ avaliações, fato que foi acentuado com a chuva registrada na $4^{\mathrm{a}}$ avaliação e que deteriorou as iscas de todos os tratamentos (Figura 3D).

Sousa (1996) menciona que a ação da chuva deteriora as iscas granuladas, da mesma forma Ferronato (2013) também cita que a ação das chuvas é o principal fator de deterioração de iscas granuladas. Burato (2013) destaca que a umidade prejudica o estado físico das iscas, deteriorando as mesmas que depois de umedecidas não são mais carregadas pelas formigas-cortadeiras, o autor também comenta que em estações do ano com menor precipitação as iscas se mantêm atrativas para as formigas por mais tempo. As observações desses autores, também foram constatadas neste estudo, pois em todas as estações do ano verificou-se que as iscas distribuídas a granel apresentaram baixa resistência a umidade.

Mariconi (1979), Mendes Filho (1981), Atta Kill (1993), Dow Elanco (1996), Andrei (1999; 2003), citam a ação da umidade como uma das principais desvantagens das iscas granuladas, pois estas, depois de 


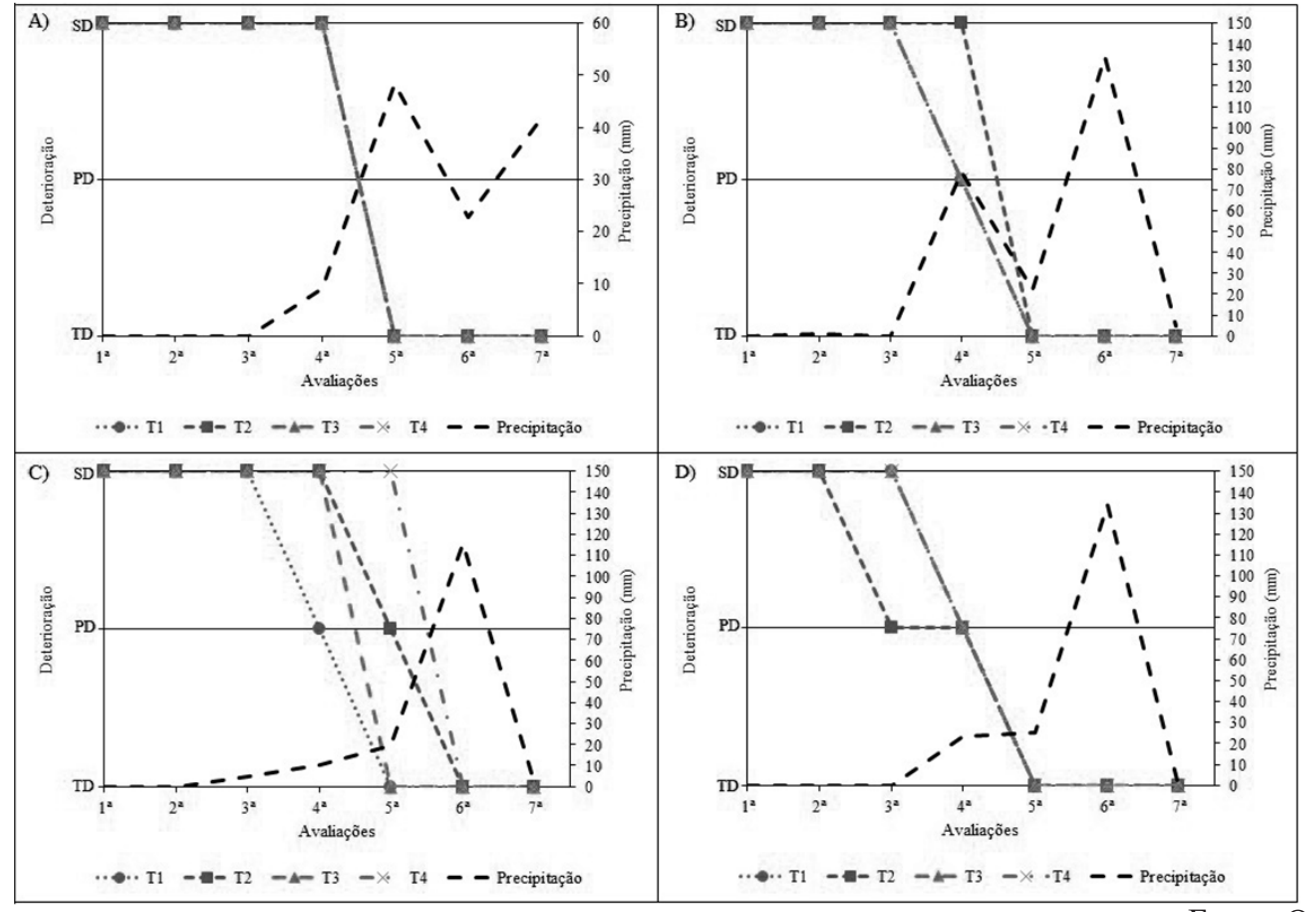

Fonte: $\mathrm{O}$ autor.

Em que: sem deterioração; PD: parcialmente deteriorada; TD: totalmente deteriorada; T1: $250 \mathrm{~g} / \mathrm{ha}$; T2: $500 \mathrm{~g} / \mathrm{ha} ; \mathrm{T} 3: 1000 \mathrm{~g} / \mathrm{ha} ; \mathrm{T} 4: 4000 \mathrm{~g} / \mathrm{ha}$.

FIGURA 3: Relação da precipitação acumulada com a conservação das iscas granuladas distribuídas a granel nas quatro estações: primavera (a); verão (b); outono (c); e inverno (d) .

FIGURE 3: Ratio of accumulated rainfall with the conservation of bulk granulated baits distributed in the four seasons: spring (a); summer (b); autumn (c); and winter (d).

úmidas perderam atratividade às formigas. Fato que foi comprovado durante as observações deste trabalho, pois não foi constatado carregamento pelas formigas-cortadeiras de iscas granuladas danificadas pela ação da umidade.

Observou-se ainda nas avaliações de campo que na primeira averiguação diária ( $8 \mathrm{~h}$ da manhã) a umidade presente no terreno (solo e plantas), originada pela neblina que é comum na região, era um fator que acelerava a deterioração física das iscas distribuídas a granel. Burato et al. (2012), em experimentos realizados na mesma região do estado de Santa Catarina e em altitudes semelhantes as avaliadas neste trabalho, também constataram que a neblina acelerava a deterioração de iscas granuladas distribuídas a granel.

\section{Influência da umidade sobre iscas granuladas distribuídas em microporta-isca}

Na primavera, os microporta-isca de todos os tratamentos ficaram conservados mesmo com o início da chuva registrada na $4^{\mathrm{a}}$ avalição. Entre a $5^{\mathrm{a}}$ e $6^{\mathrm{a}}$ avaliação, ocorreu na área experimental uma precipitação de $50 \mathrm{~mm}$, em função desta, na $6^{\mathrm{a}}$ avaliação, constatou-se que os microporta-iscas do tratamento T3 (1.000 $\mathrm{g} / \mathrm{ha})$ estavam totalmente deteriorados. Porém, nos tratamentos T1 $(250 \mathrm{~g} / \mathrm{ha}), \mathrm{T} 2(500 \mathrm{~g} / \mathrm{ha})$ e T4 (4.000 g/ ha), só foi constatada a deterioração total na $7^{\mathrm{a}}$ avaliação (Figura 4A).

No verão, observou-se que os microporta-iscas dos tratamentos T2, T3 e T4 resistiram à precipitação registrada na $4^{\mathrm{a}}$ avaliação, mantendo as iscas intactas, nos microporta-iscas do tratamento T1, constatou-se que estes estavam parcialmente deteriorados na $5^{\mathrm{a}}$ avaliação. Na $6^{\mathrm{a}}$ avaliação, os microportaiscas do tratamento T1 e T2 estavam parcialmente deteriorados, as unidades do tratamento T3 estavam 
totalmente deterioradas e as do tratamento T4 não apresentavam indícios de deterioração. $\mathrm{Na} 7^{\mathrm{a}}$ avaliação, os microporta-iscas de todos os tratamentos estavam totalmente deteriorados (Figura 4B).

No outono, as precipitações começaram a ocorrer no mesmo período da $3^{\text {a }}$ avaliação, contudo, não afetaram os microporta-iscas de todos os tratamentos, que permaneceram intactos até a $5^{\text {a }}$ avaliação. A partir da $6^{\mathrm{a}}$ avaliação observou-se deterioração parcial no tratamento $\mathrm{T} 1$ e nos demais tratamentos foi observada deterioração parcial apenas na $7^{\mathrm{a}}$ avaliação (Figura 4C).

No inverno, o início das precipitações também coincidiu com o período da $3^{\mathrm{a}}$ avaliação. Entre a $4^{\mathrm{a}}$ e a $5^{\text {a }}$ avaliações começaram a ser observados microporta-iscas parcialmente deteriorados no tratamento T3. Para os tratamentos T1, T2 e T4 constatou-se a deterioração parcial entre a $5^{\mathrm{a}}$ e a $6^{\mathrm{a}}$ observação. $\mathrm{Na} 7^{\text {a }}$ avaliação, os tratamentos $\mathrm{T} 1$ e T4 estavam totalmente deteriorados, os tratamentos T1 e T4 que permaneceram com o parâmetro de parcialmente deteriorados. (Figura 4D).

Na primavera e verão, o efeito da umidade e os danos provocados foram mais intensos do que o observado no outono e no inverno. Pois nestas estações, foi constatado o consumo parcial das iscas contidas nos microporta-iscas pelas formigas-cortadeiras Acromyrmex spp.. Esta observação também foi relatada

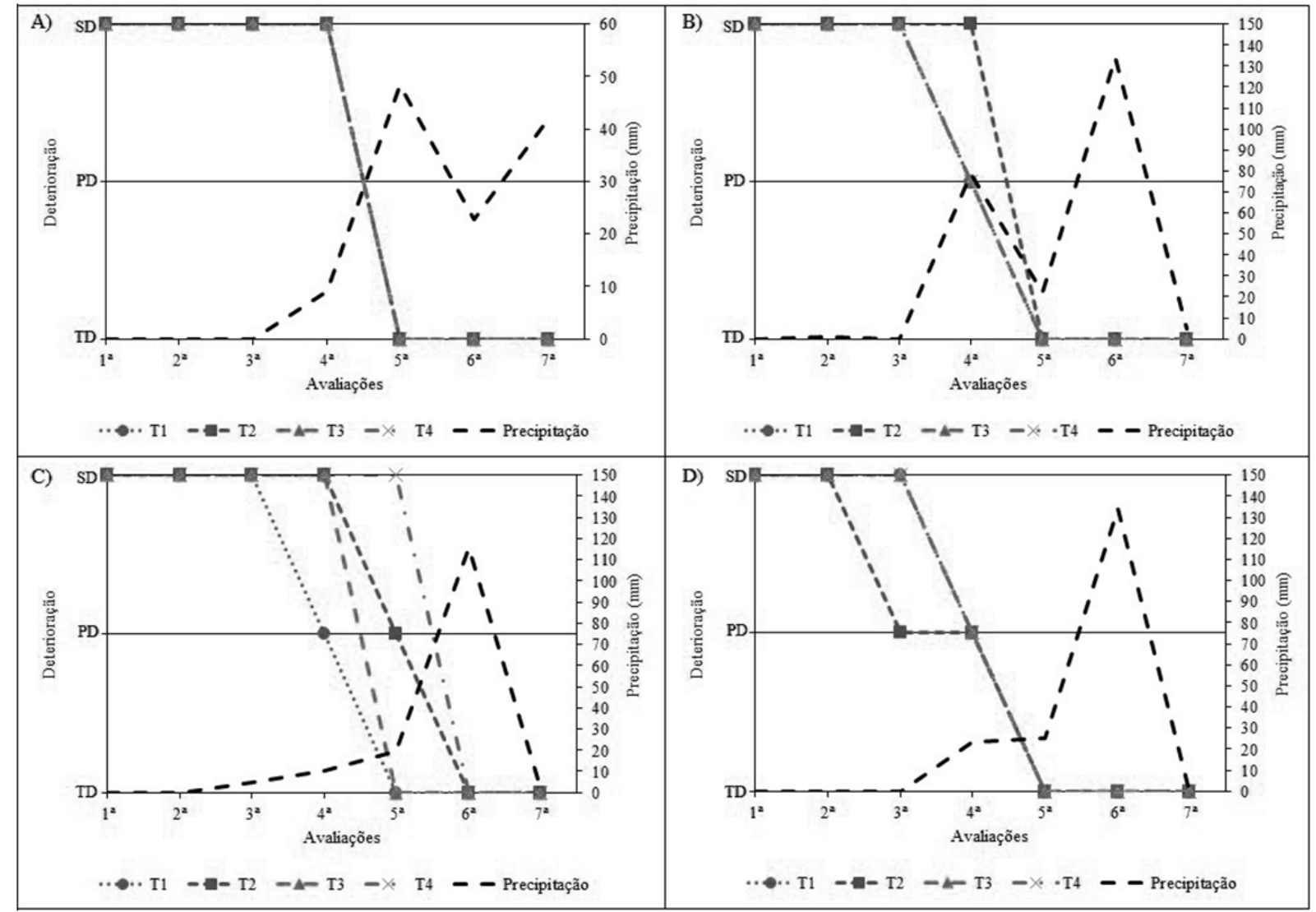

Fonte: $\mathrm{O}$ autor.

Em que: sem deterioração; PD: parcialmente deteriorada; TD: totalmente deteriorada; T1: 250 g/ha; T2: $500 \mathrm{~g} / \mathrm{ha}$; T3: $1000 \mathrm{~g} / \mathrm{ha}$; T4: $4000 \mathrm{~g} / \mathrm{ha}$

FIGURA 4: Relação da precipitação acumulada com a conservação das iscas granuladas distribuídas em microporta-iscas nas quatro estações: primavera (a); verão (b); outono (c); e inverno (d) .

FIGURE 4: Ratio of accumulated rainfall with the conservation of granulated baits distributed in bait holder the four seasons: spring (a); summer (b); autumn (c); and winter (d). 
por Sousa (1996), que em seus experimentos constatou que, quando os formigueiros de Acromyrmex spp. são pequenos, o consumo parcial das iscas é acentuado, com este consumo parcial as iscas que permanecem no microporta-iscas ficam expostas à umidade e deterioram-se com mais facilidade.

Nas estações que não houve consumo de iscas (outono e inverno), os tratamentos com microportaisca permaneceram intactos nas primeiras semanas de distribuição no campo, apresentando deterioração cerca de 30 dias após a distribuição.

Na Tabela 2, constata-se que em relação às doses, o efeito da umidade não foi o mesmo para os tratamentos com iscas granulada distribuídas a granel e que os tratamentos diferiram estatisticamente $(\mathrm{t}=$ $0,05)$, isso se devesse provavelmente ao efeito de bloco. Os blocos 3 e 4 localizavam-se em áreas mais altas e secas do talhão, em contrapartida, os blocos 1 e 2 se encontravam aproximadamente $10 \mathrm{~m}$ abaixo.

Nota-se também que o efeito da umidade foi igual para todos os tratamentos com microportaiscas, independentemente da dose utilizada no experimento. Esta constatação se deve ao fato de todas as iscas estarem igualmente protegidas por uma embalagem. No entanto, em relação aos períodos que os microporta-iscas ficaram expostos no campo, houve diferença quanto à resistência das embalagens aos efeitos da umidade.

Ferronato (2013) também observou em seus experimentos, com microporta-iscas semelhantes aos utilizados neste trabalho, que a deterioração das iscas contidas nestes recipientes está diretamente ligada à presença de orvalho e ao o aumento da precipitação nas áreas nas quais são distribuídos.

Correlacionando o consumo de iscas com a resistência a umidade dos microporta-iscas, houve diferença estatística entre a conservação de microporta-iscas em relação às estações que tiveram consumo. $\mathrm{Na}$ primavera, os microporta-iscas, mesmo sendo abertos, conservaram-se por mais tempo que no verão,

TABELA 2: Análise estatística do último registro de iscas granuladas distribuídas a granel e em microportaiscas, sem deterioração, nas quatro estações do ano.

TABLE 2: Statistical analysis of the last record of bait grainy distributed in bulk and bait holder without deterioration, the four seasons.

\begin{tabular}{|c|c|c|c|c|c|c|}
\hline \multirow{2}{*}{ Estação } & \multicolumn{4}{|c|}{ Doses distribuídas a granel $(\mathrm{g} / \mathrm{ha})$} & \multirow{2}{*}{\multicolumn{2}{|c|}{ Total $^{1}$}} \\
\hline & 250 & 500 & 1000 & 4000 & & \\
\hline Primavera & 4 & 4 & 4 & 4 & $0,69 \pm 0,00$ & A \\
\hline Verão & 3 & 4 & 3 & 3 & $0,64 \pm 0,06$ & $\mathrm{AB}$ \\
\hline Outono & 3 & 4 & 4 & 5 & $0,68 \pm 0,08$ & A \\
\hline Inverno & 3 & 2 & 3 & 3 & $0,62 \pm 0,08$ & B \\
\hline Total $^{1}$ & $0,62 \pm 0,08 \mathrm{~b}$ & $0,65 \pm 0,08 \mathrm{ab}$ & $0,67 \pm 0,04 \mathrm{ab}$ & $0,69 \pm 0,05 \mathrm{a}$ & - & \\
\hline A & & & $0,019^{*}$ & & $0,0027^{* *}$ & \\
\hline DMS & & & 05759 & & 0,05759 & \\
\hline $\mathrm{CV}(\%)$ & & & 9,20 & & & \\
\hline
\end{tabular}

\begin{tabular}{|c|c|c|c|c|c|c|}
\hline \multirow{2}{*}{ Estação } & \multicolumn{4}{|c|}{ Doses distribuídas na forma de microporta-isca (g/ha) } & \multirow{2}{*}{\multicolumn{2}{|c|}{ Total $^{1}$}} \\
\hline & 250 & 500 & 1000 & 4000 & & \\
\hline Primavera & 6 & 6 & 5 & 6 & $0,81 \pm 0,03$ & A \\
\hline Verão & 4 & 5 & 5 & 6 & $0,78 \pm 0,05$ & $\mathrm{AB}$ \\
\hline Outono & 5 & 6 & 6 & 6 & $0,84 \pm 0,05$ & A \\
\hline Inverno & 5 & 5 & 4 & 5 & $0,80 \pm 0,05$ & $\mathrm{~B}$ \\
\hline$\alpha$ & \multicolumn{4}{|c|}{$0,23^{\text {ns }}$} & \multicolumn{2}{|l|}{$0,029^{*}$} \\
\hline DMS & \multicolumn{4}{|c|}{-} & \multicolumn{2}{|l|}{0,04623} \\
\hline $\mathrm{CV}(\%)$ & \multicolumn{4}{|c|}{-} & \multicolumn{2}{|l|}{6,01} \\
\hline
\end{tabular}

Em que: Dados transformados em: $\log (\mathrm{x}+1)$; ${ }^{*}$ Significativo a $5 \%$ de probabilidade de erro; ${ }^{* *}$ Significativo a $1 \%$ de probabilidade de erro. Médias seguidas pela mesma letra maiúsculas nas colunas e minúsculas nas linhas, não diferem estatisticamente entre si, em nível de 5\% de probabilidade pelo teste de Tukey (Fonte: O autor, 2013). 
isso porque o registro de chuva na primavera foi menor, ou seja, isto comprova que a precipitação tem forte influência sobre a conservação das iscas no interior das embalagens de microporta-iscas. A porcentagem de iscas deterioradas está relacionada com o consumo parcial das iscas, pois os microporta-iscas consumidos parcialmente deixam as iscas expostas à umidade, conforme já foi comentado nos parágrafos acima.

Em relação às estações em que não houve consumo, e por consequência os microporta-iscas não foram abertos pelas formigas (outono), estes mantiveram-se mais tempo conservados a campo, devido ao registro de chuva nesta estação ter sido menor que no inverno.

\section{CONCLUSÕES}

- A umidade da região do planalto sul-catarinense degrada as iscas granuladas distribuídas a granel.

- As iscas granuladas distribuídas a granel permanecem conservadas sete dias na primavera e três dias nas demais estações do ano.

- As iscas granuladas distribuídas em microporta-iscas permanecem conservadas 15 dias no verão e 30 dias nas demais estações do ano.

- Os microporta-iscas apresentam maior resistência à umidade no campo em relação às iscas granuladas distribuídas a granel.

\section{AGRADECIMENTOS}

Ao meu orientador Nilton José Sousa. Aos professores do Programa de Pós-Graduação em Engenharia Florestal da Universidade Federal do Paraná, pelos conhecimentos transmitidos. À Florestal Rio Marombas, pelo apoio financeiro e cessão da Fazenda para instalação do experimento.

\section{REFERÊNCIAS}

ALMEIDA, A. F; ALVES, J. E. M. Controle integrado de saúvas na Aracruz Florestal. Aracruz: Aracruz Florestal, 1982. $72 \mathrm{p}$.

ANDREI, E. Compêndio de defensivos agrícolas. 6. ed. São Paulo: Saraiva, 1999.

ANDREI, E. Compêndio de defensivos agrícolas. 6. ed. compl. atual. São Paulo: Saraiva, 2003. v. 2.

ATTA-KILL. Formigas cortadeiras, problemas e soluções. Dossiê técnico. São Paulo: [s. n], 1993. 28 p. BANZATTO, D. A.; KRONKA, S. N. Experimentação Agrícola. 4. ed. Jaboticabal: Funep, 2006. 237 p.

BURATTO, D. A. et al. Avaliação dos danos causados por formigas-cortadeiras do gênero Acromyrmex (Hymenoptera) aos plantios de Pinus taeda no planalto sul-catarinense. Revista Floresta, Curitiba, v. 42, n. 4, p. 683 - 690, 2012.

BURATTO, D. A. Uso de isca granulada em plantações de Pinus taeda L., no planalto sul catarinense: avaliação de consumo por formigas cortadeiras, formas de distribuição e degradação. 2013. 72 f. Dissertação (Mestrado em Engenharia Florestal) - Universidade Federal do Paraná, Curitiba, 2013.

CHERRETT, J. M. et al. Some aspects of the development of toxic baits for the control os leaf-cutting ants. In: PROCEEDING OF THE INTERNATIONAL CONGRESS - IUSS, 7., Londres. Abstracts... Londres, 1973. p. 69-75.

DELA LUCIA, T. M. C.; VILELA, E. F. Métodos Atuais de Controle e Perspectivas. In: DELLA LUCIA, T. M. C. As formigas-cortadeiras. Viçosa: Folha de Viçosa, 1993. p. 163-190.

DOW ELANCO, L. Manual técnico de controle de formigas cortadeiras. [s. 1.: s. n.], 1996.

EMBRAPA. Centro Nacional de Pesquisa de Florestas. Zoneamento ecológico para plantios florestais no Estado de Santa Catarina. Curitiba: EMBRAPA, 1988. 113 p.

FERREIRA, D. F. SISVAR: um programa para análises e ensino de estatística. Revista Symposium, Lavras, v. 6, p. 36-41, 2008.

FERRONATO, M. Z. Avaliação de dois sistemas de distribuição de iscas granuladas, à granel e em micro-porta-iscas, no controle de formigas cortadeiras. 2013. 89 f. Dissertação (Mestrado em Engenharia Florestal) - Universidade Federal do Paraná, Curitiba, 2013.

GONÇALVES, C. R. O gênero Acromyrmex no Brasil (Hymenoptera: Formicidae). Studia Entomologica, 
Petrópolis, v. 4, n. 1/4, p. 113-180, 1961.

GONÇALVES, C. R. O gênero Acromyrmex no Brasil. 1957. 69 f. Tese (Concurso de Entomologia e Parasitologia Agrícola da Escola Nacional de Agronomia) - Universidade Rural do Rio de Janeiro, 1957. HÖLLDOBLER, B.; WILSON, E.O. The ants. Cambridge: Harvard University Press, 1990. 733 p. JURUENA, L. F. As formigas cortadeiras. Boletim de divulgação, Pelotas, n. 23, p. 19-24, 1980. LARANJEIRO, A. J. et al. Análise da distribuição de micro-porta-iscas em áreas de reforma de Eucalyptus spp., visando o controle de formigas cortadeiras (Atta spp. e Acromyrmex spp.). Piracicaba: [s.n.], 1986. 10 p.

MARICONI, F. A. M. As saúvas. São Paulo: IPEF, 1979. (Circular Técnica, n. 77).

MAYHÉ-NUNES, A. J. Estudo de Acromyrmex (Hymenoptera: Formicidae) com ocorrência constatada no Brasil: subsídios para uma análise filogenética. 1991. 122 f. Dissertação (Mestrado em Entomologia). Universidade Federal de Viçosa, Viçosa, MG, 1991.

MENDES FILHO, J. M. A. Reunião técnica de combate as formigas. Série Técnica - IPEF, Piracicaba, v. 2, n. 7, p. 19, 1981.

NICKELE, M. A. Distribuição espacial, danos e planos de amostragem de Acromyrmex crassispinus (Forel, 1909) (Hymenoptera: Formicidae: Myrmicinae) em plantios de Pinus taeda L. (Pinaceae). 2008. 125 f. Dissertação (Mestrado em Ciências Biológicas) - Universidade Federal do Paraná, Curitiba, 2008.

PACHECO, P.; REIS, W.; BRIDI, G. Verificação da aceitação e ação de iscas formicidas em diferentes períodos do ano para formigas quenquéns - resultados preliminares. In: ENCONTRO DE MIRMECOLOGIA, 15. 2001. Londrina. Anais... Londrina: IAPAR, 2001. p. 267-268.

RANDO, J. S. S.; FORTI, L. C. Ocorrência de formigas Acromyrmex Mayr, 1865, em alguns municípios do Brasil. Acta Scientiarum, Maringuá, v. 27, p. 129 - 133, 2005.

REIS FILHO, W; OLIVEIRA, S. Atividade externa, carregamento de isca granulada e Controle de Acromyrmex crassispinus em floresta de Pinus taeda. Colombo: Embrapa Florestas, 2002. 3 p.

SOUSA, N. J. Avaliação do uso de três tipos de porta-iscas no controle de formigas cortadeiras, em áreas preparadas para implantação de povoamentos de Pinus taeda L. 1996. 72 f. Dissertação (Mestrado em Engenharia Florestal) - Universidade Federal do Paraná, Curitiba, 1996.

ZANUNCIO, J. C et al. Eficiência da isca granulada Mirex-S, à base de sulfluramida, no controle de formigas cortadeira Atta laevigata (F. Smith, 1858) (HYMENOPTERA: FORMICIDAE). Revista Árvore, Viçosa, MG, v. 16, n. 3, p. 247 - 372, 1992. 